OPEN ACCESS

Edited by:

Laurel L. Lenz,

University of Colorado, United States

Reviewed by:

Werner Solbach,

Universität zu Lübeck, Germany

Sunil Joshi,

University of Miami, United States

*Correspondence:

Susan M. Bueno

sbueno@bio.puc.cl

Specialty section:

This article was submitted to

Microbial Immunology,

a section of the journal

Frontiers in Microbiology

Received: 16 May 2018

Accepted: 13 August 2018

Published: 18 September 2018

Citation:

Peñaloza HF, Noguera LP, Riedel CA and Bueno SM (2018) Expanding

the Current Knowledge About the Role of Interleukin-10 to Major Concerning Bacteria.

Front. Microbiol. 9:2047. doi: 10.3389/fmicb.2018.02047

\section{Expanding the Current Knowledge About the Role of Interleukin-10 to Major Concerning Bacteria}

\author{
Hernán F. Peñaloza' ${ }^{1}$ Loreani P. Noguera' ${ }^{1}$ Claudia A. Riedel${ }^{2}$ and Susan M. Bueno ${ }^{1 *}$ \\ ${ }^{1}$ Millennium Institute on Immunology and Immunotherapy, Departamento de Genética Molecular y Microbiología, Facultad \\ de Ciencias Biológicas, Pontificia Universidad Católica de Chile, Santiago, Chile, ${ }^{2}$ Millennium Institute on Immunology and \\ Immunotherapy, Departamento de Ciencias Biológicas, Facultad de Ciencias de la Vida, Universidad Andrés Bello, Santiago, \\ Chile
}

Interleukin-10 (IL-10) is one of the most important anti-inflammatory cytokine produced during bacterial infection. Two related phenomena explain the importance of IL-10 production in this context: first, the wide range of cells able to produce this cytokine and second, the wide effects that it causes on target cells. In a previous report we described opposing roles of IL-10 production during bacterial infection. Overall, during infections caused by intracellular bacteria or by pathogens that modulate the inflammatory response, IL-10 production facilitates bacterial persistence and dissemination within the host. Whereas during infections caused by extracellular or highly inflammatory bacteria, IL-10 production reduces host tissue damage and facilitates host survival. Given that these data were obtained using antibiotic susceptible bacteria, the potential application of these studies to multi-drug resistant (MDR) bacteria needs to be evaluated. MDR bacteria can become by 2050 a major death cause worldwide, not only for its ability to resist antimicrobial therapy but also because the virulence of these strains is different as compared to antibiotic susceptible strains. Therefore, it is important to understand the interaction of MDR-bacteria with the immune system during infection. This review discusses the current data about the role of IL-10 during infections caused by major circulating antibiotic resistant bacteria. We conclude that the production of $\mathrm{IL}-10$ improves host survival during infections caused by extracellular or highly inflammatory bacteria, however, it is detrimental during infections caused by intracellular bacteria or bacterial pathogens that modulate the inflammatory response. Importantly, during MDRbacterial infections a differential IL-10 production has been described, compared to non-MDR bacteria, which might be due to virulence factors specific of MDR bacteria that modulate production of IL-10. This knowledge is important for the development of new therapies against infections caused by these bacteria, where antibiotics effectiveness is dramatically decreasing.

Keywords: interleukin-10, multi-drug resistance, bacterial infection, immune response, host-pathogen interactions 


\section{INTRODUCTION}

Infectious diseases caused by pathogenic bacteria have been historically a major cause of human death. The discovery and mass production of antibiotics dramatically reduced the mortality associated to bacterial infections for several years. However, the golden age of antibiotic seems to be over: according to a report led by the economist Jim O'Neill, in 2017 around 700,000 deaths were caused by multi-drug resistant (MDR) bacterial infections. His prediction, however, indicates that by 2,050 the mortality associated to MDR-bacterial infections will be around of 10 million (O’Neill, 2016).

Due to this phenomenon, the World Health Organization (WHO) has classified the most concerning MDR-bacteria into different groups of priority according with the urgency to develop new antibiotics and therapies (Tacconelli, 2017). The critical priority group includes Acinetobacter baumannii, Pseudomonas aeruginosa, and Mycobacterium tuberculosis. Also, different species of the Enterobacteriaceae genus, including Klebsiella pneumoniae, Escherichia coli, are part of this group. The high priority group includes the pathogenic bacteria Staphylococcus aureus, Neisseria gonorrhoeae, Salmonella spp., among others.

In a previous report we discussed the relevance of the antiinflammatory cytokine Interleukin-10 (IL-10) in the immunity against different pathogenic bacteria (Peñaloza et al., 2016). We conclude that IL-10 production is required for host survival during infections caused by extracellular and/or highly proinflammatory bacteria, including Streptococcus pneumoniae, Pseudomonas aeruginosa, Francisella tularensis, Escherichia coli, and Mycobacterium tuberculosis (Peñaloza et al., 2016). On the other hand, IL-10 production impairs host survival during infections caused by intracellular bacteria or bacterial pathogens that modulate the inflammatory response, such as Klebsiella pneumoniae, Bordetella pertussis, Listeria monocytogenes, Brucella abortus and Salmonella enterica serovar Typhimurium (Peñaloza et al., 2016).

During extracellular or/and highly pro-inflammatory bacterial infection, the pathogen mostly neutralizes or eliminates the immune response effectors. In this context, the production of IL-10 modulates the immune response intensity and allows a successful bacterial clearance, without excessive host tissue damage. Although in some cases the absence of IL-10 makes the immune response more effective to clear the pathogen, the damage produced on host tissues is more severe and compromise host survival (Peñaloza et al., 2016).

The strategies used by intracellular pathogens and/or bacteria that modulate the inflammatory response are different and mainly consist in their capacity to evade the immune system (Bhavsar et al., 2007). In this condition, the production of IL-10 during the infective cycle of these bacteria helps them to evade the immune response and to disseminate within the host, seriously impairing host survival.

The studies performed so far that evaluate the role of IL-10 during bacterial infections have been performed using antibiotic susceptible bacteria. The conclusions reached in these reports cannot be translated to MDR-bacteria. Recent data have described that the immune response against antibiotic susceptible and MDR strains of the same bacterial specie may be remarkably different (de Breij et al., 2012; Xiong et al., 2015). Therefore, it raises two major questions about infections caused by MDR-bacteria. First: is the available data accurate enough to predict the role of immune effectors, such as anti-inflammatory cytokines, during MDR bacterial infections? And second: is it possible to develop new therapies against MDR bacteria with the current knowledge? To date, there are not enough data available to certainly answer both of these questions. However, in the last years increased efforts have made possible to understand better the role of some immune components in the response against different MDR bacteria.

In this mini-review, we will discuss whether the role of IL-10 during infections caused by major antibiotic susceptible bacteria applies to major MDR-bacterial infections. Next, we will analyze whether MDR-bacteria induce a differential production of IL-10 as compared to non-MDR bacteria, to finally discuss about the possible mechanisms that may explain this difference.

\section{THE OPPOSING ROLE OF IL-10 DURING MULTI-DRUG RESISTANT BACTERIAL INFECTION}

Carbapenem-resistant Acinetobacter baumannii (A. baumannii) is an opportunistic, aerobic, Gram-negative, and extracellular bacteria classified as a critical pathogen by the WHO (Tacconelli, 2017). A. baumannii is able to evade neutrophil mediated killing, including neutrophil extracellular traps (NETs) degradation (Kamoshida et al., 2015) or uptake inhibition after adherence (Kamoshida et al., 2015). This bacterium is commonly associated to primary health care facilities, causing skin infections, pneumonia, urinary tract infections and sepsis, mostly in immunocompromised patients (Lee et al., 2017; Harding et al., 2018).

One study demonstrated that during A. baumannii pneumonia, MDR A. baumannii virulence varies significantly as compared to non-MDR-A. baumannii (de Breij et al., 2012). Importantly, the production of IL-10 in the lungs was positively correlated with host survival (de Breij et al., 2012). Another report supported this observation (Noto et al., 2017). That report evaluated the role of receptor for advanced glycation end products (RAGE) during an A. baumannii infection. RAGE ${ }^{-/-}$ mice are more resistant to a pneumonia caused by non-MDR A. baumannii (Noto et al., 2017). The elevated survival rate displayed by $\mathrm{RAGE}^{-/-}$mice is dependent on IL-10. Given that the neutralization of this cytokine reversed the resistant phenotype observed in these mice, and at the same time the administration of recombinant IL-10 improves host survival of susceptible wild type (WT) mice (Noto et al., 2017).

Carbapenem-resistant Pseudomonas aeruginosa ( $P$. aeruginosa) is another bacterium classified in the critical group (Tacconelli, 2017). P. aeruginosa is a Gram-negative opportunistic pathogenic bacterium able to causes chronic 
respiratory tract infections in patients with cystic fibrosis and in patients with compromised immune system (Gellatly and Hancock, 2013). Due to the importance of $P$. aeruginosa, since long time ago scientists have started to use clinical isolates to study host-pathogen interaction, specifically ampicillin resistant strains (Chmiel et al., 1999, 2002; Parker et al., 2016; Riquelme et al., 2017). A recent study analyzed cytokine production, cellular recruitment and bacterial clearance during the first $12 \mathrm{~h}$ during an intraperitoneal infection with four MDR isolates, three non-MDR isolates and two reference strains of $P$. aeruginosa in mice (Gomez-Zorrilla et al., 2017a). MDR isolates induced a stronger immune response able to clear more efficiently the infective bacteria at 12 hpi (Gomez-Zorrilla et al., 2017a). These data help to understand the differences of virulence and pathogenicity between MDR and not-MDR strains. However, given that the infection route was intraperitoneally and not intranasal, we cannot conclude whether the production of IL-10 is beneficial or detrimental during pneumonia caused by MDR $P$. aeruginosa in this particular experiment. Despite that, all the evidences show that IL-10 production protects the host during P. aeruginosa.

Both Carbapenem-resistant and $3^{\text {rd }}$ generation of cephalosporin resistant Enterobacteriaceae have also been categorized in the critical group (Tacconelli, 2017). Within this group are included Klebsiella pneumonia, Escherichia coli, Enterobacter spp., Serratia spp., Proteus spp., Providencia spp. and Morganella spp. Most of the studies performed on these bacteria are epidemiological (Liñares et al., 2010; Rojas et al., 2017), genetic (Deleo et al., 2014; Rojas et al., 2017), or have been focused to understand the mechanisms involved in the acquisition of antibiotic resistance (Fuzi et al., 2017; Dhar et al., 2018). However, few studies have been focused in the analysis of host-pathogen interaction with MDR-strains. Regarding to that these reports describe the

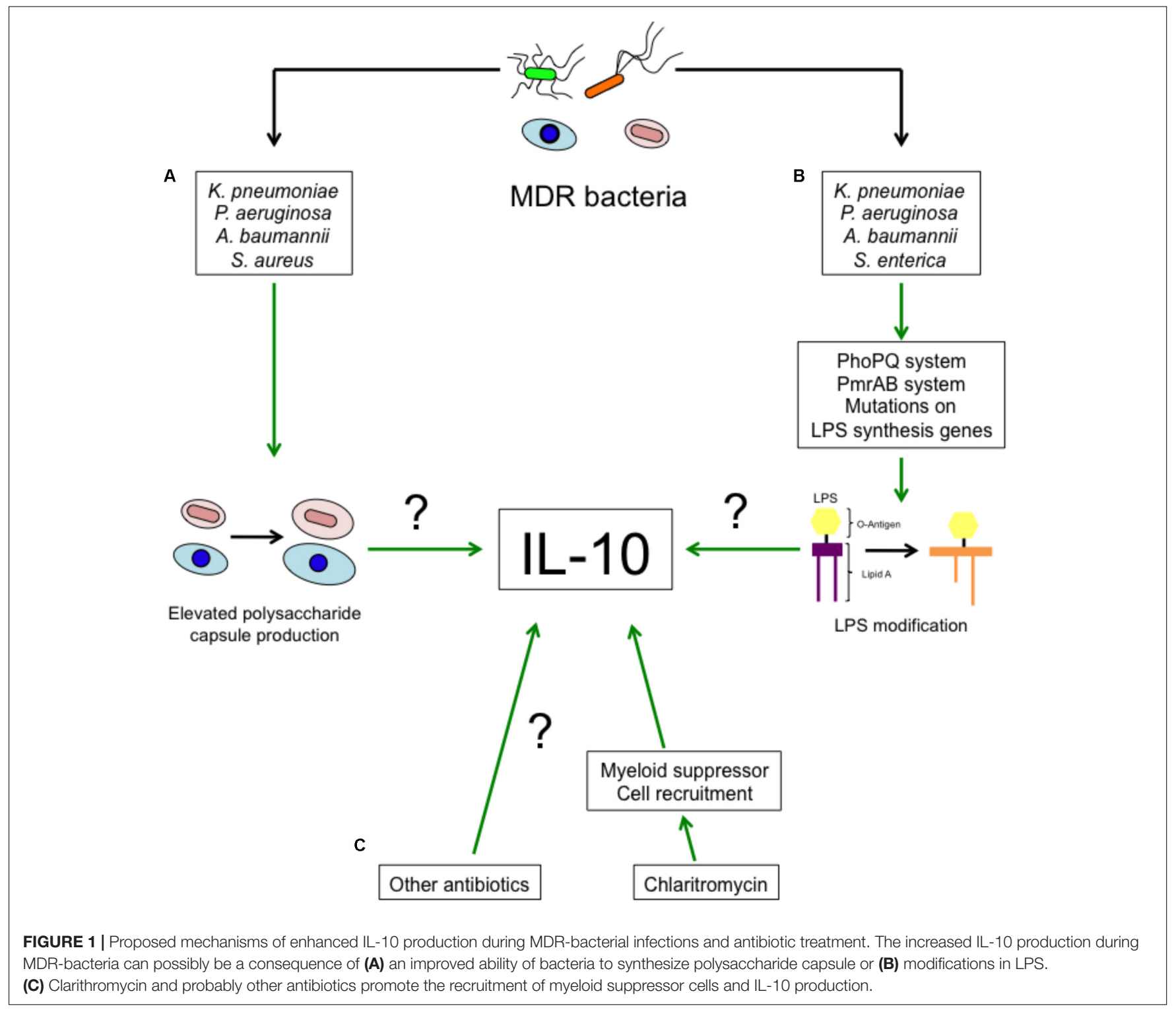


immune response against carbapenem-resistant $K$. pneumoniae (CRKP) (Tzouvelekis et al., 2013; Xiong et al., 2015; Ahn et al., 2016). These studies showed that CRKP isolates are less virulent (Tzouvelekis et al., 2013; Xiong et al., 2015) than antibiotic susceptible $K$. pneumoniae strains, but resistant to neutrophil-mediated clearance (Tzouvelekis et al., 2013; Ahn et al., 2016; Kobayashi et al., 2016). CRKP showed to be highly susceptible to human serum (Olonisakin et al., 2016; DeLeo et al., 2017) and the anti-capsule antibodies generation helped to innate cells to clear this pathogen in vivo (Kobayashi et al., 2018). Another important characteristic of CRKP is the primary role of monocytes in the pathogenesis caused by this bacterium. For monocytes, at least two different roles have been described during CRKP infection: some of the monocytes recruited to the lungs are Monocytic Myeloid-derived cells (M-MDSCs) (Ahn et al., 2016) probably able to establish an anti-inflammatory environment, aiding CRKP to evade the immune response. On the other hand, inflammatory monocytes are essential to phagocyte and kill CRKP as well as to activate ILC3s and $\mathrm{Th}_{17}$ response (Xiong et al., 2016).

Mycobacterium tuberculosis ( $M$. tuberculosis) is another bacterium included in the critical WHO group. M. tuberculosis is a rod-shaped bacterium with a cell wall rich in lipids able to cause chronic and latent infections in the lung tissue (Glickman and Jacobs, 2001; Bañuls et al., 2015). During nonMDR M. tuberculosis infections, IL-10 production by neutrophils is important for host survival (Peñaloza et al., 2016). But there is not data available that describe the immunity and the role of IL-10 during MDR M. tuberculosis infection at molecular level.

Methicillin/Vancomycin-resistant Staphylococcus aureus and 3rd generation cephalosporin/fluoroquinolone-resistant Neisseria gonorrhoeae (N. gonorrhoeae) are the only WHO high priority bacterial species for which the role of IL-10 production has been described (Liu et al., 2013; Leech et al., 2017). Staphylococcus aureus is an extracellular Gram-positive bacterium able to cause serious lung (Kitur et al., 2015) and skin infections (Kitur et al., 2016). Most of host-pathogen interaction research has been done on Methicillin-resistant $S$. aureus (MRSA), and the result of this research is the identification of bacterial and host factors involved in MRSA infection (Parker et al., 2016; Leech et al., 2017).

During a MRSA (USA300) infection there is a rapid production of IL-10 (Leech et al., 2017). The contribution of this cytokine for host survival varies according the nature of the infection. During an acute systemic infection, the production of IL-10 by $\mathrm{CD} 19^{+} \mathrm{CDB} 220^{+}$cells was required to control the bacterial dissemination and its production is associated with host survival (Leech et al., 2017). However, in a sub-cutaneous infection model, IL-10 produced mostly by MDSCs in the local wound impairs MRSA clearance, without affecting host survival (Leech et al., 2017). MRSA infects orthopedic devices. In this case, the production of IL-10 by MDSCs favors the biofilm formation on the surface of these devices inducing persistent infections that do not affect host mortality (Heim et al., 2014, 2015).
Neisseria gonorrhoeae is an obligate Gram-negative human pathogen (Balthazar et al., 2011; Araneta et al., 2017) causative of gonorrhea, a sexually transmitted disease (Balthazar et al., 2011; Araneta et al., 2017). During an intra-vaginal N. gonorrhoeae infection, the neutralization of IL-10 through the administration of microspheres improved the clearance of $N$. gonorrhoeae and enhanced the polarization to a $\mathrm{Th}_{1}$ response (Liu et al., 2013). These findings suggest that the production of IL-10 is detrimental for the immunity against this bacterium (Liu et al., 2013).

Fluoroquinolone-resistant Salmonella enterica (S. enterica) is a pathogenic bacterium categorized in the high priority group with S. aureus and N. gonerrhoeae (Tacconelli, 2017). $S$. enterica is a Gram-negative foodborne pathogenic bacterium (Lamas et al., 2018). Similar to other bacteria, few host-pathogen interaction studies have been reported for MDR Salmonella and yet the role of IL-10 during MDR Salmonella infection remains unknown. However, we have recently reported the role of IL-10 during an infection with non-MDR-Salmonella enterica serovar Typhimurium (Salazar et al., 2017). This report demonstrates that IL-10 produced mostly by $\mathrm{T}$ cells impairs Salmonella clearance and increases host mortality (Salazar et al., 2017). Further research is needed to evaluate whether MDR-S. Typhimurium will differentially induce IL-10 production during infection.

\section{MDR AND NON-MDR BACTERIA INDUCE DIFFERENTIAL IL-10 PRODUCTION DURING INFECTION}

In the previous section, we described that the production of IL10 plays a critical role during MDR-bacterial infections. Similar to infections caused by non-MDR bacteria, the production of IL-10 may lead to a better or worse outcome depending of the infecting bacteria. Since the MDR-bacteria and non-MDR bacteria present different virulence, it is possible to hypothesize that MDR-bacteria induce differential amounts of IL-10 during infection. Indeed, several studies corroborate this hypothesis. For example, ICU patients infected with extensively drugresistant (XDR) $P$. aeruginosa presented elevated amounts of IL-10 in blood, as compared to patients infected with nonMDR P. aeruginosa (Gomez-Zorrilla et al., 2017b). Same result was obtained in a mouse model of $P$. aeruginosa pneumonia (Tam et al., 2018). Consistently, patients infected with MDR $M$. tuberculosis presented higher amounts of IL-10 in the serum, as compared to patients infected with non-MDR M. tuberculosis (Pinheiro et al., 2013). However, another group did not find any difference in IL-10 production in patients infected with MDR or non-MDR M. tuberculosis (Eum et al., 2008). Moreover, during a $S$. aureus endocarditis model, rabbits infected with MRSA presented higher amounts of IL-10 as compared to those infected with Methicillin susceptible $S$. aureus in the serum (Tsaganos et al., 2013).

All these data suggest that MDR-bacteria might induce more IL-10 production than non-MDR bacteria. The only exception was found in A. baumannii infection, where children infected 


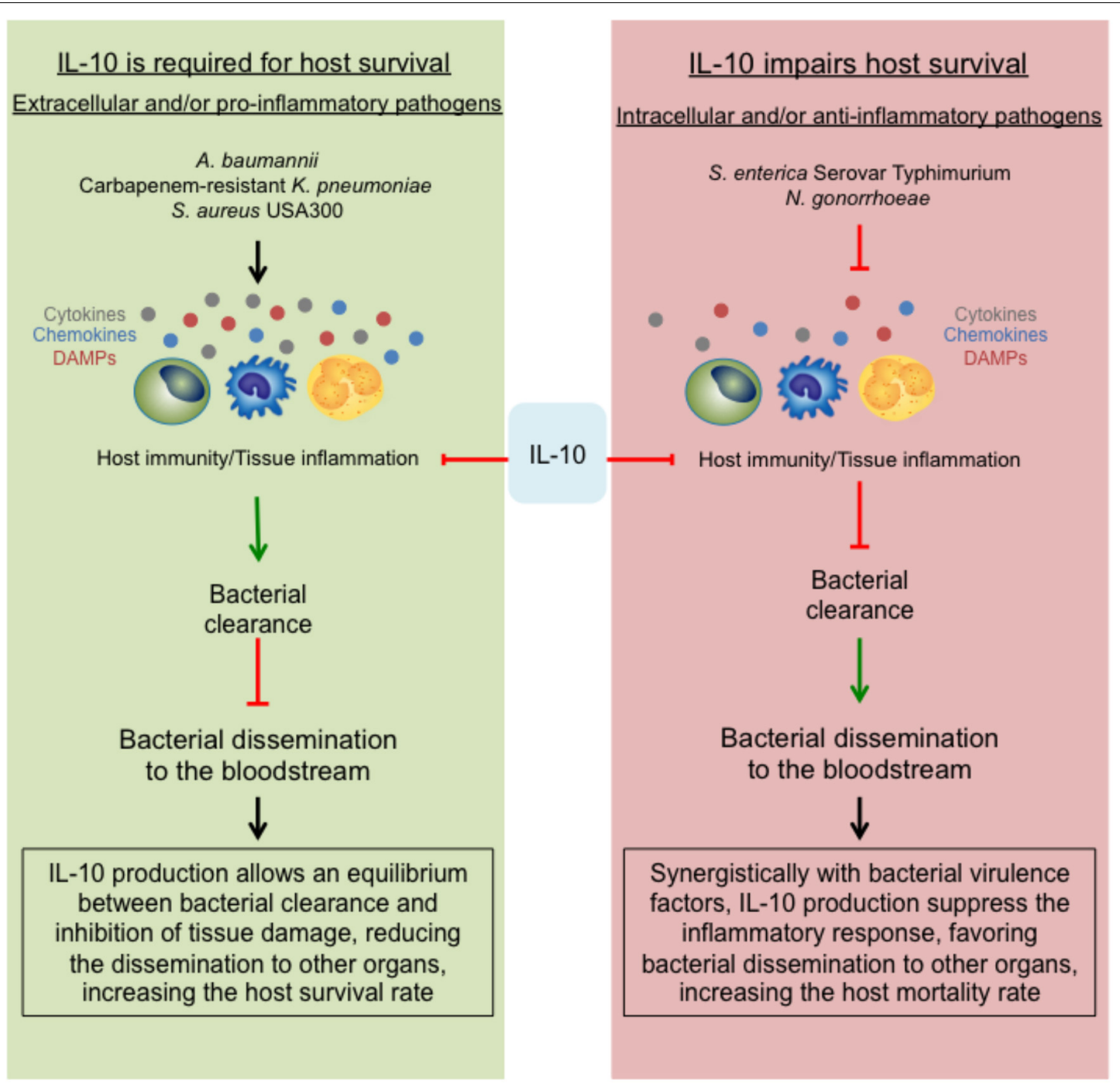

FIGURE 2 | The opposing role of the anti-inflammatory effect of IL-10. During MDR bacterial infections, the suppressive role of IL-10 can lead into two different outcomes. (Left) Describes that during infections with extracellular and/or pro-inflammatory bacteria, the strong immune response will be essentially modulated by IL-10, allowing a slow clearance with limited host tissue damage, generating an equilibrium required for host survival. On the contrary, during infections with intracellular and/or anti-inflammatory bacteria (right), the production of IL-10 together with the bacterial virulence strategies will shut down the immune response, impairing the bacterial clearance, facilitating the dissemination within the host and at the end, increasing the host mortality rate.

either with MDR A. baumannii presented the same levels of IL-10 as compared with children infected with non-MDR A. baumannii (Fang et al., 2016).

The next question then is as to how MDR-bacteria induce higher levels of IL-10. It is well known that antibiotic resistance not only involves the acquisition of hydrolytic enzymes, but also includes changes in proteins structure or expression of genes involved in virulence such as porins, two-component systems and others (Beceiro et al., 2013). Polysaccharide capsule (Figure 1A), present in bacteria such as $K$. pneumoniae, $P$. aeruginosa, A. baumannii, S. aureus, among other bacteria, is an important determinant for antimicrobial peptides and Polymyxin B resistance (Llobet et al., 2008). An increased ability to produce capsule may be an adaptation against antibiotics. Interestingly, capsule recognition by pattern recognition receptors (PRRs) triggers IL-10 production in myeloid cells (Peñaloza et al., 2016). MgrB is an important regulator of gene expression in $K$. pneumoniae, deletion of $m g r B$ increases the resistance to collistin and Polymyxin B by the remodeling of lipid A mediated by the PhoPQ system (Kidd et al., 2017). This increased resistance is associated with increased virulence and reduced TNF- $\alpha$ production (Kidd et al., 2017). Lipid A remodeling has also been observed in MDR P. aeruginosa, also dependent on the activity of the PhoPQ system (Barrow and Kwon, 2009; Lee et al., 2017). PmrAB, another two-component system, is also involved in LPS modification and promotes antibiotic resistance in $S$. enterica and P. aeruginosa (Gunn et al., 2000; Barrow and Kwon, 2009). Similarly, A. baumannii resistance to Polymyxin B is 
mediated by mutations in $l p x A, l p x C$ and $l p x D$. These mutations lead to the loss of LPS by altering the biosynthesis of the lipid A (Moffat et al., 2010) and induce a weaker immune response. It is unknown whether LPS modification by MDR-bacteria leads to higher production of IL-10. This possibility is highly probable, given that LPS recognition by TLR4 induces IL-10 production in myeloid cells (Figure 1B) (Peñaloza et al., 2016).

Multi-drug resistant $N$. gonorrhoeae carries the opa gene (Hess et al., 2012). Opa inhibits the proliferation of $\mathrm{CD} 4^{+} \mathrm{T}$ cells and $B$ cells in mice (Boulton and Gray-Owen, 2002), increases the expression of PD-L1 and stimulates the production of IL-10 (Zhu et al., 2012).

Even though the treatments against MDR bacteria are limited, they mostly consist of antibiotic administration. A recent report describe that Clarithromycin promotes the recruitment of antiinflammatory myeloid cells able to produce IL-10, Arginase-1 and iNOS to the lung tissue and the spleen (Namkoong et al., 2018). The recruitment of these cells to the lung protects the host during pneumococcal pneumonia and in LPS endotoxin shock (Namkoong et al., 2018). It is unknown whether other antibiotics also induce the production of IL-10 or other anti-inflammatory mediators. This would be an interesting hypothesis to evaluate (Figure 1C).

\section{CONCLUSION}

In the last years, increasing data support the idea that IL10 production drives the development of a successful immune response during bacterial infection. However, whether IL-10 production is beneficial or not for host survival depends of the pathogen nature and the immune response associated to the infection (Peñaloza et al., 2016).

The rapid emergence of MDR bacteria is a major concern and has pushed the research community to invest more efforts in studying these bacteria. Most of the research done on MDR bacteria has been focused on epidemiological and genetic characterization (Liñares et al., 2010; Deleo et al., 2014; Rojas et al., 2017); and limited numbers of studies have been focused in the understanding of host-pathogen interaction. The most important reason for the existence of this gap is the high amount of data available regarding non-MDR strains and the assumption that virulence and pathogenicity of MDR and non-MDR bacteria are similar. Recent reports show that pathogenicity and virulence can be remarkably different between MDR and non-MDR strains (de Breij et al., 2012; Tzouvelekis et al., 2013; Xiong et al., 2015).

The data discussed in this mini-review support the dual role of IL-10, previously described for non-MDR can also be found during MDR bacterial infection. For non-MDR bacterial infection IL-10 production is required for host survival during infections caused by extracellular and/or highly proinflammatory bacteria. Conversely, for MDR bacterial infection, IL-10 impairs host survival and bacterial clearance during intracellular and/or weak pro-inflammatory bacteria. During A. baumannii and MRSA infection, the production of IL10 is required for host survival. Both bacteria have acquired different virulence factors that allow them to neutralize the host immune response at different stages. In this scenario, a hyperactive immune response may occur, being IL-10 essential to modulate the intensity of it (Figure 1, left panel).

As we discussed previously, most of the studies about hostpathogen interaction of $P$. aeruginosa has been done using ampicillin resistant strains. In this scenario the production of IL10 seems to be required for host survival, however, whether these data can be translated to carbapenem-resistant $P$. aeruginosa is unknown and more studies need to be done.

S. enterica and $N$. gonorrhoeae are both intracellular pathogens, either way; IL-10 absence and/or neutralization improve the host immunity against these microbes and increase host survival (Figure 2, right panel). Overall, these data is consistent with our initial hypothesis.

Carbapenem-resistant K. pneumoniae is a special case. $K$. pneumoniae is an extracellular bacterium that induces a weak pro-inflammatory response (Ahn et al., 2016). Published data show that during non-MDR $K$. pneumoniae infection, IL-10 production facilitates bacterial dissemination and impairs host survival (Peñaloza et al., 2016). Several reports show that the virulence and pathogenicity of MDR strains are reduced as compared to non-MDR strains (Tzouvelekis et al., 2013; Xiong et al., 2015; Ahn et al., 2016). It is quite remarkable that even though CRKP doses typically used in mice studies are around $1 \times 10^{8} \mathrm{CFUs} /$ mice, WT C57BL/6J are highly resistant to these infections (Tzouvelekis et al., 2013; Xiong et al., 2015; Ahn et al., 2016). These bacteria are also resistant to neutrophil-mediated clearance (Ahn et al., 2016; Kobayashi et al., 2016) and during CRKP there is a rapid peak of IL-10 production (Ahn et al., 2016). Taking account all this data, we hypothesize that during CRKP infection IL-10 is a major immune response modulator required for host survival (Figure 2, left panel).

One of the most important concerns of this report is the lack of research regarding host-pathogen interaction on MDR bacteria. It is clear by the whole community that the golden age of antibiotics is over, however, the knowledge of how these bacteria have adapted to the host immune response and how we can improve it is very limited. Therefore, we believe that more research in host immunity and bacterial virulence is required to design new strategies to identify effective therapeutic targets or strategies.

Finally, it is quite interesting MDR-bacteria stimulates higher IL-10 production as compared with non-MDR bacteria. We believe that the differential production of IL-10 may be a consequence of the changed expression pattern of different virulence factors displayed by these bacteria. This fact could explain why in general these bacteria are less virulent and why higher bacterial dose are commonly used to study host-pathogen interaction in mice.

\section{AUTHOR CONTRIBUTIONS}

All authors listed have made substantial, direct, and intellectual contribution to the work and approved it for publication. 


\section{FUNDING}

This study was supported by Fondo Nacional de Desarrollo Científico y Tecnológico de Chile (FONDECYT) \#1170964 and \#1161525, Millennium Institute on

\section{REFERENCES}

Ahn, D., Peñaloza, H., Wang, Z., Wickersham, M., Parker, D., Patel, P., et al. (2016). Acquired resistance to innate immune clearance promotes Klebsiella pneumoniae ST258 pulmonary infection. JCI Insight 1:e89704. doi: 10.1172/jci. insight.89704

Araneta, C. M. P., Juayang, A. C., Lim, J. P. T., Quilop, E. M. G., Casaysay, N. J. G., Tamesis, G. M. L., et al. (2017). Antibiotic susceptibility monitoring of Neisseria gonorrhoeae in bacolod city, philippines. Trop. Med. Infect. Dis. 2:45. doi: 10.3390/tropicalmed2030045

Balthazar, J. T., Gusa, A., Martin, L. E., Choudhury, B., Carlson, R., and Shafer, W. M. (2011). Lipooligosaccharide structure is an important determinant in the resistance of Neisseria gonorrhoeae to antimicrobial agents of innate host defense. Front. Microbiol. 2:30. doi: 10.3389/fmicb.2011.00030

Bañuls, A. L., Sanou, A., Anh, N. T., and Godreuil, S. (2015). Mycobacterium tuberculosis: ecology and evolution of a human bacterium. J. Med. Microbiol. 64, 1261-1269. doi: 10.1099/jmm.0.000171

Barrow, K., and Kwon, D. H. (2009). Alterations in two-component regulatory systems of phoPQ and pmrAB are associated with polymyxin B resistance in clinical isolates of Pseudomonas aeruginosa. Antimicrob. Agents Chemother. 53, 5150-5154. doi: 10.1128/AAC.00893-09

Beceiro, A., Tomas, M., and Bou, G. (2013). Antimicrobial resistance and virulence: a successful or deleterious association in the bacterial world? Clin. Microbiol. Rev. 26, 185-230. doi: 10.1128/CMR.00059-12

Bhavsar, A. P., Guttman, J. A., and Finlay, B. B. (2007). Manipulation of hostcell pathways by bacterial pathogens. Nature 449, 827-834. doi: 10.1038/ nature 06247

Boulton, I. C., and Gray-Owen, S. D. (2002). Neisserial binding to CEACAM1 arrests the activation and proliferation of CD4+ T lymphocytes. Nat. Immunol. 3, 229-236. doi: 10.1038/ni769

Chmiel, J. F., Konstan, M. W., Knesebeck, J. E., Hilliard, J. B., Bonfield, T. L., Dawson, D. V., et al. (1999). IL-10 attenuates excessive inflammation in chronic Pseudomonas infection in mice. Am. J. Respir. Crit. Care Med. 160, 2040-2047. doi: 10.1164/ajrccm.160.6.9901043

Chmiel, J. F., Konstan, M. W., Saadane, A., Krenicky, J. E., Lester Kirchner, H., and Berger, M. (2002). Prolonged inflammatory response to acute Pseudomonas challenge in interleukin-10 knockout mice. Am. J. Respir. Crit. Care Med. 165, 1176-1181. doi: 10.1164/ajrccm.165.8.2107051

de Breij, A., Eveillard, M., Dijkshoorn, L., van den Broek, P. J., Nibbering, P. H., and Joly-Guillou, M. L. (2012). Differences in Acinetobacter baumannii strains and host innate immune response determine morbidity and mortality in experimental pneumonia. PLoS One 7:e30673. doi: 10.1371/journal.pone. 0030673

Deleo, F. R., Chen, L., Porcella, S. F., Martens, C. A., Kobayashi, S. D., Porter, A. R., et al. (2014). Molecular dissection of the evolution of carbapenem-resistant multilocus sequence type 258 Klebsiella pneumoniae. Proc. Natl. Acad. Sci. U.S.A. 111, 4988-4993. doi: 10.1073/pnas.1321364111

DeLeo, F. R., Kobayashi, S. D., Porter, A. R., Freedman, B., Dorward, D. W., Chen, L., et al. (2017). Survival of carbapenem-resistant Klebsiella pneumoniae sequence type 258 in human blood. Antimicrob. Agents Chemother. 61:e0253316. doi: 10.1128/AAC.02533-16

Dhar, S., Kumari, H., Balasubramanian, D., and Mathee, K. (2018). Cell-wall recycling and synthesis in Escherichia coli and Pseudomonas aeruginosa - their role in the development of resistance. J. Med. Microbiol. 67, 1-21. doi: 10.1099/ jmm.0.000636

Eum, S. Y., Jeon, B. Y., Min, J. H., Kim, S. C., Cho, S., Park, S. K., et al. (2008). Tumor necrosis factor-alpha and interleukin-10 in whole blood is associated with disease progression in pulmonary multidrug-resistant tuberculosis patients. Respiration 76, 331-337. doi: 10.1159/000113932
Immunology and Immunotherapy P09/016-F, Grant \#13CTI-21526 P5 from INNOVA-CORFO program of Chilean Ministry of Economy, and Comisión Nacional de Investigación Científica y Tecnológica (CONICYT) \#21140214 (HP).

Fang, C., Chen, X., and Zhou, M. (2016). Epidemiology and cytokine levels among children with nosocomial multidrug-resistant Acinetobacter baumannii complex in a tertiary hospital of Eastern China. PLoS One 11:e0161690. doi: 10.1371/journal.pone.0161690

Fuzi, M., Szabo, D., and Csercsik, R. (2017). Double-serine fluoroquinolone resistance mutations advance major international clones and lineages of various multi-drug resistant bacteria. Front. Microbiol. 8:2261. doi: 10.3389/fmicb.2017. 02261

Gellatly, S. L., and Hancock, R. E. (2013). Pseudomonas aeruginosa: new insights into pathogenesis and host defenses. Pathog. Dis. 67, 159-173. doi: 10.1111/ 2049-632X.12033

Glickman, M. S., and Jacobs, W. R. Jr. (2001). Microbial pathogenesis of Mycobacterium tuberculosis: dawn of a discipline. Cell 104, $477-485$.

Gomez-Zorrilla, S., Calatayud, L., Juan, C., Cabot, G., Tubau, F., Oliver, A., et al. (2017a). Understanding the acute inflammatory response to Pseudomonas aeruginosa infection: differences between susceptible and multidrug-resistant strains in a mouse peritonitis model. Int. J. Antimicrob. Agents 49, 198-203. doi: 10.1016/j.ijantimicag.2016.10.016

Gomez-Zorrilla, S., Morandeira, F., Castro, M. J., Tubau, F., Periche, E., Canizares, R., et al. (2017b). Acute Inflammatory response of patients with Pseudomonas aeruginosa infections: a prospective study. Microb. Drug Resist. 23, 523-530. doi: 10.1089/mdr.2016.0144

Gunn, J. S., Ryan, S. S., Van Velkinburgh, J. C., Ernst, R. K., and Miller, S. I. (2000). Genetic and functional analysis of a PmrA-PmrB-regulated locus necessary for lipopolysaccharide modification, antimicrobial peptide resistance, and oral virulence of Salmonella enterica serovar typhimurium. Infect. Immun. 68, 6139-6146.

Harding, C. M., Hennon, S. W., and Feldman, M. F. (2018). Uncovering the mechanisms of Acinetobacter baumannii virulence. Nat. Rev. Microbiol. 16, 91-102. doi: 10.1038/nrmicro.2017.148

Heim, C. E., Vidlak, D., and Kielian, T. (2015). Interleukin-10 production by myeloid-derived suppressor cells contributes to bacterial persistence during Staphylococcus aureus orthopedic biofilm infection. J. Leukoc. Biol. 98, 1003-1013. doi: 10.1189/jlb.4VMA0315-125RR

Heim, C. E., Vidlak, D., Scherr, T. D., Kozel, J. A., Holzapfel, M., Muirhead, D. E., et al. (2014). Myeloid-derived suppressor cells contribute to Staphylococcus aureus orthopedic biofilm infection. J. Immunol. 192, 3778-3792. doi: 10.4049/ jimmunol.1303408

Hess, D., Wu, A., Golparian, D., Esmaili, S., Pandori, W., Sena, E., et al. (2012). Genome sequencing of a Neisseria gonorrhoeae isolate of a successful international clone with decreased susceptibility and resistance to extendedspectrum cephalosporins. Antimicrob. Agents Chemother. 56, 5633-5641. doi: 10.1128/AAC.00636-12

Kamoshida, G., Kikuchi-Ueda, T., Tansho-Nagakawa, S., Nakano, R., Nakano, A., Kikuchi, H., et al. (2015). Acinetobacter baumannii escape from neutrophil extracellular traps (NETs). J. Infect. Chemother. 21, 43-49. doi: 10.1016/j.jiac. 2014.08.032

Kidd, T. J., Mills, G., Sa-Pessoa, J., Dumigan, A., Frank, C. G., Insua, J. L., et al. (2017). A Klebsiella pneumoniae antibiotic resistance mechanism that subdues host defences and promotes virulence. EMBO Mol. Med. 9, 430-447. doi: 10.15252/emmm.201607336

Kitur, K., Parker, D., Nieto, P., Ahn, D. S., Cohen, T. S., Chung, S., et al. (2015). Toxin-induced necroptosis is a major mechanism of Staphylococcus aureus lung damage. PLoS Pathog. 11:e1004820. doi: 10.1371/journal.ppat.1004820

Kitur, K., Wachtel, S., Brown, A., Wickersham, M., Paulino, F., Peñaloza, H. F., et al. (2016). Necroptosis promotes Staphylococcus aureus clearance by inhibiting excessive inflammatory signaling. Cell Rep. 16, 2219-2230. doi: 10.1016/j.celrep. 2016.07.039 
Kobayashi, S. D., Porter, A. R., Dorward, D. W., Brinkworth, A. J., Chen, L., Kreiswirth, B. N., et al. (2016). Phagocytosis and killing of carbapenemresistant ST258 Klebsiella pneumoniae by human neutrophils. J. Infect. Dis. 213, 1615-1622. doi: 10.1093/infdis/jiw001

Kobayashi, S. D., Porter, A. R., Freedman, B., Pandey, R., Chen, L., Kreiswirth, B. N., et al. (2018). Antibody-mediated killing of carbapenem-resistant ST258 Klebsiella pneumoniae by human neutrophils. MBio 9:e00297-18. doi: 10.1128/ mBio.00297-18

Lamas, A., Miranda, J. M., Regal, P., Vazquez, B., Franco, C. M., and Cepeda, A. (2018). A comprehensive review of non-enterica subspecies of Salmonella enterica. Microbiol. Res. 206, 60-73. doi: 10.1016/j.micres.2017.09.010

Lee, C. R., Lee, J. H., Park, M., Park, K. S., Bae, I. K., Kim, Y. B., et al. (2017). Biology of Acinetobacter baumannii: pathogenesis, antibiotic resistance mechanisms, and prospective treatment options. Front. Cell Infect. Microbiol. 7:55. doi: 10.3389/fcimb.2017.00055

Leech, J. M., Lacey, K. A., Mulcahy, M. E., Medina, E., and McLoughlin, R. M. (2017). IL-10 plays opposing roles during Staphylococcus aureus systemic and localized infections. J. Immunol. 198, 2352-2365. doi: 10.4049/jimmunol. 1601018

Liñares, J., Ardanuy, C., Pallares, R., and Fenoll, A. (2010). Changes in antimicrobial resistance, serotypes and genotypes in Streptococcus pneumoniae over a 30-year period. Clin. Microbiol. Infect. 16, 402-410. doi: 10.1111/j.14690691.2010.03182.x

Liu, Y., Egilmez, N. K., and Russell, M. W. (2013). Enhancement of adaptive immunity to Neisseria gonorrhoeae by local intravaginal administration of microencapsulated interleukin 12. J. Infect. Dis. 208, 1821-1829. doi: 10.1093/ infdis/jit354

Llobet, E., Tomas, J. M., and Bengoechea, J. A. (2008). Capsule polysaccharide is a bacterial decoy for antimicrobial peptides. Microbiology 154(Pt 12), 3877-3886. doi: 10.1099/mic.0.2008/022301-0

Moffat, H., Harper, M., Harrison, P., Hale, J. D., Vinogradov, E., Seemann, T., et al. (2010). Colistin resistance in Acinetobacter baumannii is mediated by complete loss of lipopolysaccharide production. Antimicrob. Agents Chemother. 54, 4971-4977. doi: 10.1128/AAC.00834-10

Namkoong, H., Ishii, M., Fujii, H., Yagi, K., Asami, T., Asakura, T., et al. (2018). Clarithromycin expands CD11b $+\mathrm{Gr}-1+$ cells via the STAT3/Bv8 axis to ameliorate lethal endotoxic shock and post-influenza bacterial pneumonia. PLoS Pathog. 14:e1006955. doi: 10.1371/journal.ppat.1006955

Noto, M. J., Becker, K. W., Boyd, K. L., Schmidt, A. M., and Skaar, E. P. (2017). RAGE-mediated suppression of interleukin-10 results in enhanced mortality in a murine model of Acinetobacter baumannii Sepsis. Infect. Immun. 85:e095416. doi: 10.1128/IAI.00954-16

Olonisakin, T. F., Li, H., Xiong, Z., Kochman, E. J., Yu, M., Qu, Y., et al. (2016). CD36 provides host protection against Klebsiella pneumoniae intrapulmonary infection by enhancing lipopolysaccharide responsiveness and macrophage phagocytosis. J. Infect. Dis. 214, 1865-1875. doi: 10.1093/infdis/jiw451

O’Neill, J. (2016). Tackling drug-resistant infections globally: final report and recommendations. Rev. Antimicrob. Resist. 1-84. Available at: https://amrreview.org

Parker, D., Ahn, D., Cohen, T., and Prince, A. (2016). Innate immune signaling activated by MDR bacteria in the airway. Physiol. Rev. 96, 19-53. doi: 10.1152/ physrev.00009.2015

Peñaloza, H. F., Schultz, B. M., Nieto, P. A., Salazar, G. A., Suazo, I., Gonzalez, P. A., et al. (2016). Opposing roles of IL-10 in acute bacterial infection. Cytokine Growth Factor Rev. 32, 17-30. doi: 10.1016/j.cytogfr.2016.07.003

Pinheiro, R. O., de Oliveira, E. B., Dos Santos, G., Sperandio da Silva, G. M., de Andrade Silva, B. J., Teles, R. M., et al. (2013). Different immunosuppressive mechanisms in multi-drug-resistant tuberculosis and nontuberculous mycobacteria patients. Clin. Exp. Immunol 171, 210-219. doi: 10. $1111 /$ cei. 12007

Riquelme, S. A., Hopkins, B. D., Wolfe, A. L., DiMango, E., Kitur, K., Parsons, R., et al. (2017). Cystic fibrosis transmembrane conductance regulator attaches tumor suppressor PTEN to the membrane and promotes anti Pseudomonas aeruginosa immunity. Immunity 47, 1169.e7-1181.e7. doi: 10.1016/j.immuni. 2017.11.010

Rojas, L. J., Weinstock, G. M., De La Cadena, E., Diaz, L., Rios, R., Hanson, B. M., et al. (2017). An analysis of the epidemic of Klebsiella pneumoniae carbapenemase-producing $K$. pneumoniae: convergence of two evolutionary mechanisms creates the "perfect storm". J. Infect. Dis. 217, 82-92. doi: 10.1093/ infdis/jix524

Salazar, G. A., Peñaloza, H. F., Pardo-Roa, C., Schultz, B. M., Munoz-Durango, N., Gomez, R. S., et al. (2017). Interleukin-10 production by $\mathrm{t}$ and b cells is a key factor to promote systemic Salmonella enterica serovar typhimurium infection in mice. Front. Immunol. 8:889. doi: 10.3389/fimmu.2017.00889

Tacconelli, E. (2017). Global Priority List of Antibiotic-Resistant Bacteria to Guide Research, Discovery, and Development of New Antibiotics. Essential Medicines and Health Products. Geneva: World Health Organization, 1-7.

Tam, V. H., Perez, C., Ledesma, K. R., and Lewis, R. E. (2018). Transcriptional profiles of pulmonary innate immune responses to isogenic antibioticsusceptible and multidrug-resistant Pseudomonas aeruginosa. Microbiol. Immunol. 62, 291-294. doi: 10.1111/1348-0421.12581

Tsaganos, T., Pelekanou, A., Skiadas, I., and Giamarellos-Bourboulis, E. J. (2013). Differences in cytokine stimulation between methicillin-susceptible and methicillin-resistant Staphylococcus aureus in an experimental endocarditis model. J. Infect. Chemother. 19, 272-278. doi: 10.1007/s10156-0120497-1

Tzouvelekis, L. S., Miriagou, V., Kotsakis, S. D., Spyridopoulou, K., Athanasiou, E., Karagouni, E., et al. (2013). KPC-producing, multidrug-resistant Klebsiella pneumoniae sequence type 258 as a typical opportunistic pathogen. Antimicrob. Agents Chemother. 57, 5144-5146. doi: 10.1128/AAC.01052-13

Xiong, H., Carter, R. A., Leiner, I. M., Tang, Y. W., Chen, L., Kreiswirth, B. N., et al. (2015). Distinct contributions of neutrophils and CCR2 + monocytes to pulmonary clearance of different Klebsiella pneumoniae strains. Infect. Immun. 83, 3418-3427. doi: 10.1128/IAI.00678-15

Xiong, H., Keith, J. W., Samilo, D. W., Carter, R. A., Leiner, I. M., and Pamer, E. G. (2016). Innate lymphocyte/Ly6C(hi) monocyte crosstalk promotes Klebsiella Pneumoniae clearance. Cell 165, 679-689. doi: 10.1016/j.cell.2016. 03.017

Zhu, W., Ventevogel, M. S., Knilans, K. J., Anderson, J. E., Oldach, L. M., McKinnon, K. P., et al. (2012). Neisseria gonorrhoeae suppresses dendritic cell-induced, antigen-dependent CD4 T cell proliferation. PLoS One 7:e41260. doi: 10.1371/journal.pone.0041260

Conflict of Interest Statement: The authors declare that the research was conducted in the absence of any commercial or financial relationships that could be construed as a potential conflict of interest.

Copyright (C) 2018 Peñaloza, Noguera, Riedel and Bueno. This is an open-access article distributed under the terms of the Creative Commons Attribution License (CC BY). The use, distribution or reproduction in other forums is permitted, provided the original author(s) and the copyright owner(s) are credited and that the original publication in this journal is cited, in accordance with accepted academic practice. No use, distribution or reproduction is permitted which does not comply with these terms. 